\title{
MMP2 and MMP10 Polymorphisms Are Related to Steroid-Induced Osteonecrosis of the Femoral Head among Chinese Han Population
}

\author{
Ye Tian, ${ }^{1,2}$ Feimeng An,, Jiaqi Wang, ${ }^{1,2}$ Chang Liu, ${ }^{1,2}$ Huiqiang Wu, ${ }^{3}$ Yuju Cao, ${ }^{4}$ \\ Jianzhong Wang $\left(\mathbb{C},{ }^{2}\right.$ and Guoqiang Wang $\mathbb{(}^{2}$ \\ ${ }^{1}$ Inner Mongolia Medical University, Hohhot, Inner Mongolia, China \\ ${ }^{2}$ Department of Trauma Orthopedics, The Second Affiliated Hospital of Inner Mongolia Medical University, Hohhot, \\ Inner Mongolia, China \\ ${ }^{3}$ Inner Mongolia Autonomous Region Hospital of Traditional Chinese Medicine, Hohhot, Inner Mongolia, China \\ ${ }^{4}$ Zhengzhou Traditional Chinese Medicine (TCM) Traumatology Hospital, Zhengzhou, Henan Province, China
}

Correspondence should be addressed to Jianzhong Wang; wangjianzhong0503@126.com

and Guoqiang Wang; wangguoqianggq@163.com

Received 26 October 2018; Accepted 26 March 2019; Published 5 May 2019

Academic Editor: Yuan Yang

Copyright (C) 2019 Ye Tian et al. This is an open access article distributed under the Creative Commons Attribution License, which permits unrestricted use, distribution, and reproduction in any medium, provided the original work is properly cited.

\begin{abstract}
Background. Steroid-induced osteonecrosis of the femoral head is a relatively serious condition which seriously reduces patient quality of life. However, the pathogenesis of steroid-induced ONFH is still unclear. In recent years, more scholars have found that the pathogenesis of steroid-induced ONFH is related to susceptibility factors such as MMPs/TIMPs system. The main purpose of this study is to investigate the correlation between MMP2 and MMP10 gene polymorphisms and steroid-induced ONFH in Chinese Han population. Methods. Six SNPs in MMP2 and two SNPs in MMP10 were genotyped using Agena MassARRAY RS1000 system from 286 patients of steroid-induced ONFH and in 309 healthy controls. The association between MMP2 and MMP10 polymorphisms and steroid-induced ONFH risk were estimated by the Chi-squared test, genetic model analysis, haplotype analysis, and stratification analysis. The relative risk was estimated by odd ratios (ORs) and $95 \%$ confidence intervals (CIs). Result. We found that the minor TG allele of rs 470154 in $M M P 10$ was associated with an increased risk of steroid-induced ONFH $(\mathrm{OR}=1.45,95 \% \mathrm{CI}$, $1.03-2.05, p=0.032$ ). In the genetic model analysis, we found that rs2241146 in MMP2 gene and rs470154 in MMP10 gene showed a statistically significant association with increased risk of steroid-induced ONFH. The six SNPs (rs470154, rs243866, rs243864, rs865094, rs11646643, and rs2241146) showed a statistically significant association with different clinical phenotypes. Conclusion. Our results verify that genetic polymorphisms of $M M P 2$ and $M M P 10$ contribute to steroid-induced ONFH susceptibility in the population of Chinese Han population, and our study provides new insights into the role that MMP2 and MMP10 plays in the mechanism of ONFH.
\end{abstract}

\section{Introduction}

Osteonecrosis of the femoral head (ONFH) is a debilitating bone disease, characterized by collapse of the femoral head and subsequent loss of hip joint function $[1,2]$. ONFH frequently occurs in young and middle-aged people and seriously affects the quality of life for patients. At present, the number of new patients diagnosed with ONFH each year is 150,000 to 200,000 in China [3]. Widespread use of corticosteroids is widely believed to be one of the common causes of nontraumatic osteonecrosis. Steroidinduced ONFH accounts for $24.1 \%$ of all femoral head necrosis [4]. In clinical practice, we can often find that not all patients with a few months of steroid administration will develop steroid-induced ONFH, suggesting that genetic factors may confer susceptibility or resistance to steroidinduced ONFH. The certain genetic background has been shown related to ONFH in previous studies; single nucleotide polymorphism (SNP) is believed to be positively correlated with steroid-induced ONFH risk $[5,6]$. 
Matrix metalloproteinases (MMPs), an enzyme family that is involved in the degradation of various proteins in the extracellular matrix (ECM), play an important part in the tissue remodeling process and physiological and pathological repair [7-9]. A complete set of MMPs produced by human cells is defined based on the complete human genome sequence. Recent genomic studies have shown that the MMP family has 24 distinct genetically encoded members, including $M M P 2$ and $M M P 10$ [10]. $M M P 2$, encoded by $M M P 2$ gene in chromosome $16 \mathrm{q} 12.2$, is a $72-\mathrm{kDa}$ type IV collagenase. $M M P 2$ is a rich enzyme secreted by osteoblasts and there is a significant negative correlation between MMP2 levels and bone mineral density [11]. Keiichi Inoue's previous experiments demonstrated that $M M P 2$ is important to the progress of forming and maintaining the osteocyte canalicular network and its formation is a determinant of bone remodeling and mineralization [12,13]. In addition, studies have shown that osteolysis can be caused by abnormal expression of $M M P 2$ [14]. In the course of ONFH, articular cartilage degenerates in the early stages of ONFH before significant radiological changes occur in the femoral head; ONFH articular cartilage degradation indicators include MMP2 [15]. MMP2 activates various cytokines and chemokines in inflammatory response [16]. Another gene $M M P 10$, encoded by $M M P 10$ gene in chromosome 11q22.2, is a secreted metalloproteinase involved in the physiological process of bone growth and wound healing [17]. In the occurrence of periodontitis, MMP10 may increase the risk of periodontitis; its mechanism is consistent with the local inflammatory response [18].

Studies have shown that the lack of functional MMP10 may be involved in the calcification process in vivo and in vitro VSMC, leading to a decrease in the degree of calcification. This result was consistent with reports of impaired atherosclerotic calcification in MMP2-deficient mice [17]. In addition, Duits et al. demonstrated higher plasma $M M P 2$ and CSF MMP10 levels in $\mathrm{AD}$ patients compared to $\mathrm{VaD}$ patients and controls [19]. In previous study, investigator found the association between genetic polymorphisms of MMP2 and the risk of alcohol-induced osteonecrosis of the femoral head in the population of China [20]. Although MMP2 has been studied more extensively than MMP10, CSF MMP10, also known as lysostromin-2, has been linked to inflammation. However, there are no reports on the association between genetic polymorphisms of MMP2 and MMP10 and the risk of steroid-induced ONFH. Given the crucial role of $M M P 2$ and $M M P 10$ in the inflammatory response, bone metabolism, and bone loss, we speculated that the polymorphism of $M M P 2$ and MMP10 might be associated with the risk of steroidinduced ONFH. Thus, we undertook the case-control study included 286 patients with steroid-induced ONFH and 309 healthy controls to elucidate the role of MMP2 and MMP10 gene polymorphisms in the occurrence of steroid-induced ONFH among Chinese Han population.

\section{Materials and Methods}

2.1. Study Participants. From September 2014 to January 2016, a total of 595 subjects included 286 patients with steroid-induced ONFH and 309 healthy controls were consecutively recruited from Zhengzhou Traditional Chinese Medicine (TCM) Traumatology Hospital. The controls were enrolled based on a physical examination, with no steroidinduced ONFH or other related diseases. All subjects were Han people living in or near Zhengzhou City.

The inclusion criteria of cases were as follows: ONFH was diagnosed based on MRI and CT scanning. Steroid-induced osteonecrosis was diagnosed based on a history of taking a mean daily dose of $16.6 \mathrm{mg}$ or an equivalent maximum daily dose of $80 \mathrm{mg}$ of prednisolone within 1 year [21]. The exclusion criteria of cases were as follows: patients did not meet the diagnostic criteria of steroid-induced ONFH; patients with traumatic ONFH, dislocation of the hip joint, or other hip diseases were excluded; patients drank more than $400 \mathrm{ml}$ per week or had significant familial hereditary disease.

This study complied with the principles of the Declaration of Helsinki. The Ethical Committee of Zhengzhou Traditional Chinese Medicine Traumatology Hospital agreed to the study and all participants provided written informed consent.

2.2. SNPs Selection and Genotyping. Based on data from the database (dbSNP and GWASs), eight SNPs of MMP2 and MMP10 were selected. All SNPs we selected had minor allele frequencies $>5 \%$ in the global population from the 1000 Genome HapMap database. We collected blood samples into EDTA tubes after centrifugation at $2000 \mathrm{rpm}$ for 10 minutes. Blood samples kept at $-80^{\circ} \mathrm{C}$ for future experiments. Genomic DNA was obtained from whole blood using GoldMag extraction method (GoldMag, China) and then stored at $-20^{\circ} \mathrm{C}$. We evaluated DNA quantity using spectrophotometry (DU530UV/VIS spectrophotometer, Beckman Instruments, Fullerton, CA, USA). Agena MassARRAY Assay Design 4.0 Software was implemented to develop the Multiplexed SNP Mass EXTEND assay. Agena MassARRAY RS1000 system was carried out to measure the SNP genotypes by the manufacturer's protocol. Data management and analyses were performed using the Agena Bioscience Typer 4.0 software.

2.3. Statistical Analyses. Statistical analyses were performed using the SPSS 20.0 software (SPSS, Chicago, IL). The Chi-square test was used to check whether the genotype distribution matched Hardy-Weinberg equilibrium (HWE) in the control group. In this study, all the tests were twotailed, and $p$ values less than 0.05 were regarded as statistically significant. We used Chi-squared test to calculate the distribution differences of allele, genotype, and haplotype. We analyzed associations between the genotypes of MMP2 and MMP10 polymorphisms and the risk of steroid-induced ONFH through different genetic models, codominant, dominant, recessive, and log-additive models by computing odds ratios (ORs) and 95\% confidence intervals (CIs) from logistic regression adjusted for age and gender. The stratification analysis was performed with clinical stages, hip lesions, and course condition. Finally, haploview software (version 4.2) was used to estimate the linkage disequilibrium (LD) and haplotype construction. 
TABLE 1: Characteristics of the individuals in controls and steroidinduced ONFH patients.

\begin{tabular}{lccc}
\hline Variables & $\begin{array}{c}\text { Cases }(\mathrm{n}=286) \\
\mathrm{N}(\%)\end{array}$ & $\begin{array}{c}\text { Controls }(\mathrm{n}=309) \\
\mathrm{N}(\%)\end{array}$ & $p$ value \\
\hline Age, years & & & \\
Mean \pm SD & $41.83 \pm 13.12$ & $48.78 \pm 8.30$ & $<0.001^{\mathrm{a}}$ \\
$\leq 48$ & $203(71.0)$ & $170(55.0)$ & \\
$>48$ & $83(29.0)$ & $139(45.0)$ & \\
Gender & & & \\
female & $113(39.5)$ & $112(36.2)$ & \\
male & $173(60.5)$ & $197(63.8)$ & \\
Clinical stages & & & \\
Stage II & $64(22.4)$ & & \\
Stage III & $126(44.1)$ & & \\
Stage IV & $96(33.6)$ & & \\
Hip lesions & & \\
Unilateral & $83(29.0)$ & \\
Bilateral & $203(71.0)$ & \\
Course, months & & \\
$>12$ & $157(45.1)$ & \\
$\leq 12$ & $129(54.9)$ & \\
\hline$P<0.05$ in & & \\
\hline
\end{tabular}

$P<0.05$ indicates statistical significance.

${ }^{\mathrm{a}}$ Independent samples $\mathrm{t}$ test.

${ }^{\mathrm{b}}$ Two-sided Chi-squared test.

\section{Result}

A total of 286 patients with steroid-induced ONFH (mean age: $41.83 \pm 13.12$ ) and 309 healthy controls (mean age: 48.78 \pm 8.30 ) were recruited for our study. Basic characteristics, including gender, age, and the clinical phenotypes (unilateral or bilateral hip lesions, $>12$ months or $\leq 12$ months course, and II, III, IV clinical stages of steroid-induced ONFH) were listed in Table 1. Steroid-induced ONFH cases and healthy controls were matched in sex $(p=0.412)$. However, we found the cases and healthy controls were not matched for age $(p<0.05)$, so we adjusted the age factor in the subsequent analysis.

Table 2 summarizes basic information about the candidate in the eight SNPs. None of the SNPs that were excluded deviated from Hardy-Weinberg equilibrium (HWE). The $\chi^{2}$ test was utilized to calculate the differences in frequency distribution of alleles between cases and controls. We found that rs470154 in MMP10 was linked to an increased risk of steroid-induced ONFH (OR $=1.45,95 \%$ CI: $1.03-2.05, p=$ 0.035).

Subsequently, we evaluated the association between these SNPs and steroid-induced ONFH risk using four genetic models (codominant, dominant, recessive, and log-additive) by logistic-regression analysis. Table 3 showed that rs 2241146 in the $M M P 2$ gene was associated with an increased risk of steroid-induced ONFH in the recessive model before (OR $=2.15,95 \%$ CI: $1.05-4.41, p=0.032$ ) and after (adjusted OR $=2.42,95 \%$ CI: $1.14-5.14, p=0.018$ ) adjustment. Moreover, rs470154 in MMP10 polymorphism was correlated with an increased risk of steroid-induced ONFH in the log-additive model before adjustment for age and gender $(\mathrm{OR}=1.44,95 \%$ CI: $1.02-2.02, p=0.035$ ).

Next, cases were stratified in terms of unilateral or bilateral hip lesions, $>12$ months or $\leq 12$ months course, and clinical stages. We further evaluated the effects of alleles and genotypes of SNPs on the risk of steroid-induced ONFH among the different subgroups (Table 4). In the allele and genotype models, the locus associated with steroid-induced risk of ONFH in patients with unilateral femoral head necrosis was rs 243866 (adjusted OR $=0.40,95 \%$ CI: $0.20-0.80, p$ $=0.007 ; p=0.013$ ), rs243864 (adjusted OR $=0.40,95 \%$ CI: $0.20-0.78, p=0.006 ; p=0.010$ ), and rs865094 (adjusted OR $=1.56,95 \%$ CI: $1.08-2.24, p=0.016 ; p=0.029)$. We found that rs11646643 and rs2241146 were only associated with steroidinduced ONFH risk in patients with unilateral femoral head necrosis in the allele model. Among them, rs2241146 was associated with steroid-induced femoral head necrosis in the genotype model (unilateral, $p=0.038$; aged $>12$ months, $p=$ 0.013). Moreover, in the allele model, rs 470154 was associated with steroid-induced ONFH risk in patients with bilateral femoral head necrosis (adjusted OR $=1.50,95 \%$ CI: 1.04-2.18, $p=0.031$ ), clinical stage III (adjusted OR $=1.76,95 \%$ CI: $1.17-2.67 ; p=0.007), \leq 12$ months course (adjusted $\mathrm{OR}=$ 1.71, 95\% CI: $1.13-2.59, p=0.010)$. In the genotype model, it was related to clinical stage III $(p=0.015)$. In general, the SNPs with protective factors were rs243866, rs243864, and rs11646643. Separately, the SNPs with increase risk were rs470154, 865094, and rs2241146. In the case-control study, we performed a power analysis of selected MMP2 and MMP10 SNPs (Table 5).

Finally, the parameter $\mathrm{D}^{\prime}$ was used to analyze the extent of linkage disequilibrium between SNPs. Then the haplotype LD block was determined according to the control group data (Figure 1). Two blocks were detected by haplotype analysis in the MMP2 gene (Figure 1), one block comprising rs243866 and rs243864 and the other one comprising rs11646643, rs2241146, and rs9928731. However, we did not find any association between the haplotype in the MMP2 and steroidinduced ONFH risk (Tables 6 and 7).

\section{Discussion}

In this study, we found rs2241146 and rs470154 were significantly associated with an increased risk of steroid-induced ONFH. Six SNPs showed a statistically significant association with different clinical phenotypes (rs470154, rs243866, rs243864, rs865094, rs11646643, and rs2241146). To our knowledge, this is the first report to investigate an association between these loci and steroid-induced ONFH susceptibility.

MMP2 has been taken into account as a target candidate gene of genetic association studies for numerous human diseases. Robert Wojciechowski et al. [22] study showed that rs9928731 in MMP2 was significantly associated with ocular refraction in the AMISH families, which implicated $M M P 2$ as regulator of nonpathologic refractive error. Ou Liu et al. [23] found that the MMP2 haplotype rs2241145-rs9928731 showed the significant association with Thoracic Aortic dissection (TAD), which may indicate the genetic predisposition to TAD in Chinese Han population. Rs9928731, rs11646643, and 
TABLE 2: Basic information of candidate SNPs in this study.

\begin{tabular}{|c|c|c|c|c|c|c|c|c|c|c|}
\hline \multirow{3}{*}{$\begin{array}{l}\text { SNP } \\
\text { rs243866 }\end{array}$} & \multirow{3}{*}{$\begin{array}{c}\text { Gene } \\
M M P 2\end{array}$} & \multirow{3}{*}{$\begin{array}{c}\text { Position } \\
16 q 12.2\end{array}$} & \multirow{3}{*}{$\begin{array}{c}\text { Alleles } \\
\text { A/B } \\
\text { A/G }\end{array}$} & \multicolumn{2}{|c|}{ MAF } & \multirow{3}{*}{$\begin{array}{c}p^{\mathrm{a}} \text { value } \\
\text { for HWE } \\
0.329\end{array}$} & \multirow{3}{*}{$\begin{array}{l}\text { ORs } \\
0.79\end{array}$} & \multirow{2}{*}{\multicolumn{2}{|c|}{$95 \% \mathrm{CI}$}} & \multirow{3}{*}{$\frac{p^{\mathrm{b}} \text { value }}{0.194}$} \\
\hline & & & & \multirow{2}{*}{$\frac{\text { case }}{0.112}$} & \multirow{2}{*}{$\begin{array}{c}\text { control } \\
0.173\end{array}$} & & & & & \\
\hline & & & & & & & & 0.56 & 1.13 & \\
\hline rs243864 & MMP2 & $16 \mathrm{q} 12.2$ & $\mathrm{G} / \mathrm{T}$ & 0.112 & 0.140 & 0.342 & 0.78 & 0.55 & 1.10 & 0.151 \\
\hline rs865094 & MMP2 & $16 \mathrm{q} 12.2$ & G/A & 0.290 & 0.266 & 1.000 & 1.13 & 0.87 & 1.45 & 0.357 \\
\hline rs11646643 & $M M P 2$ & $16 \mathrm{q} 12.2$ & G/A & 0.175 & 0.205 & 0.601 & 0.83 & 0.62 & 1.11 & 0.202 \\
\hline rs2241146 & MMP2 & $16 \mathrm{q} 12.2$ & $\mathrm{~A} / \mathrm{G}$ & 0.234 & 0.213 & 0.611 & 1.13 & 0.86 & 1.48 & 0.388 \\
\hline rs9928731 & $M M P 2$ & $16 \mathrm{q} 12.2$ & $\mathrm{~T} / \mathrm{C}$ & 0.486 & 0.476 & 0.170 & 1.04 & 0.83 & 1.31 & 0.719 \\
\hline rs470154 & $M M P 10$ & $11 \mathrm{q} 22.2$ & $\mathrm{~T} / \mathrm{G}$ & 0.149 & 0.107 & 1.000 & 1.45 & 1.03 & 2.05 & $0.032^{\star}$ \\
\hline rs17293607 & MMP10 & $11 \mathrm{q} 22.2$ & G/A & 0.003 & 0.005 & 1.000 & 0.72 & 0.12 & 4.32 & 0.931 \\
\hline
\end{tabular}

SNP: single nucleotide polymorphism, HWE: Hardy-Weinberg equilibrium, OR: odds ratio, 95\% CI: 95\% confidence interval, and MAF: minor allele frequency.

${ }^{a} P$ values were calculated by exact test.

${ }^{\mathrm{b}} P$ values were calculated by Pearson Chi-squared test.

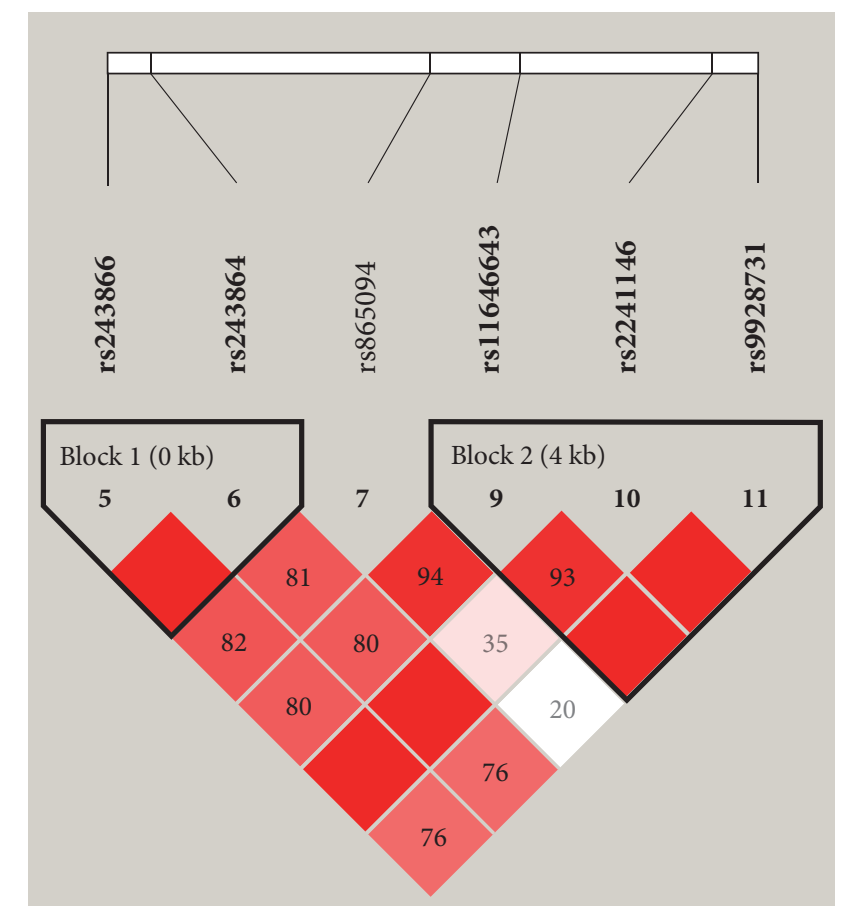

FIGURE 1: Linkage disequilibrium (LD) plots containing six SNPs from MMP2. Red squares display statistically significant associations between a pair of SNPs, as measured by D'; darker shades of red indicate higher D'.

rs2241146 have a strong linkage between each pair of the three SNPs. In our study, however, there was no association between rs9928731 and steroid-induced ONFH. Inconsistent data observed between single-label analysis and haplotype analysis may indicate that rs9928731 was not independently associated with ONFH risk, but rather the SNPs in combination. Rs 9928731 is located on chr16 of the MMP2 intron region between the sixth and seventh exons in $M M P 2$, it does not represent a coding variant and located near the region where acetylation of histone H3 lysine 27 is significantly enriched, which is a reliable chromatin marker of active enhancers. Noncoding variants in these regions can result in damage to regulatory functions, and in some cases are supposed to cause disease phenotypes. In addition, epigenetic regulation of gene expression by histone modifications is celltype specific. In the relevant region, H3 lysine 27 acetylation is highly enriched in human fibroblasts (MMP2 region) and human keratinocytes ( $M M P 1$ through $M M P 10$ intergenic region); SNPs are also possible [22, 24].

Juiz et al. [25] indicated that rs243866 may be involved in the ECM remodeling. Additionally, previous studies showed that A allele carriers may be related to decreased risk of systolic heart failure in a Han Chinese population [26]. MMP2 rs243866 is located in 5 to a half palindromic potential estrogen receptor binding site. The $1575 \mathrm{G}$ allele acts as an enhancer in estrogen receptor-positive MCF-7 cells, while the $1575 \mathrm{~A}$ allele reduces transcriptional activity. Gel migration assays confirmed that the binding of the estrogen 


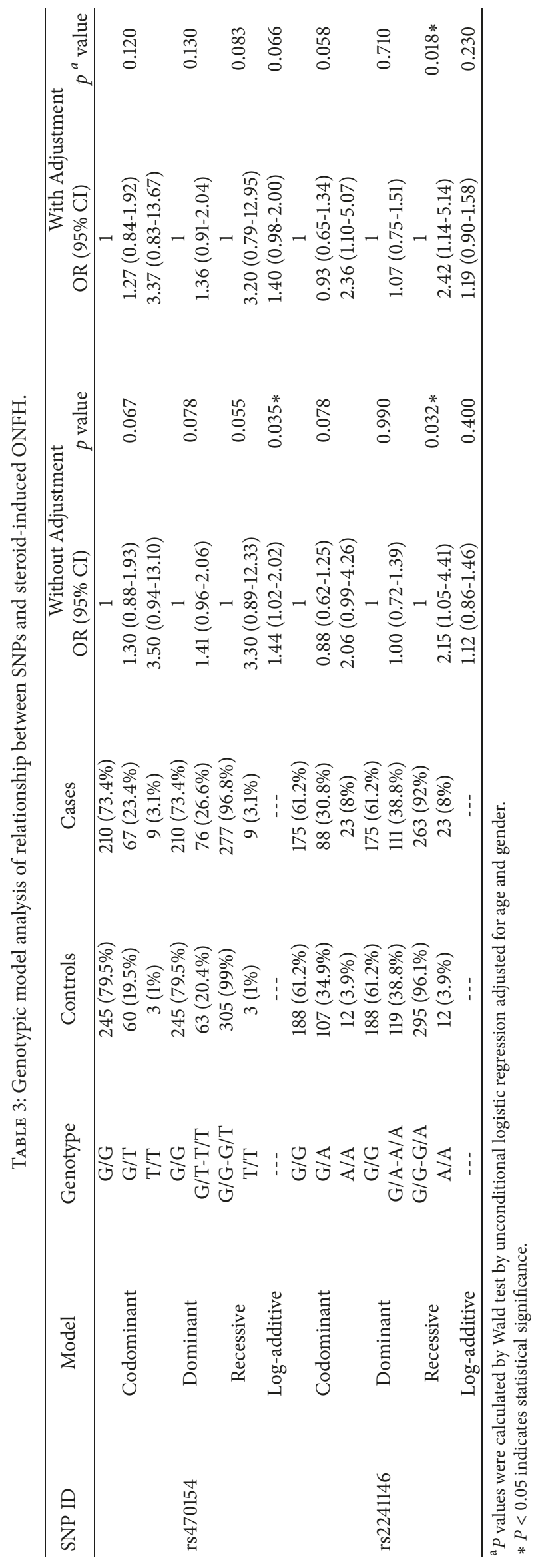


TABLE 4: Stratified analysis for association of MMP2 and MMP10 gene polymorphism with the clinical phenotypes of steroid-induced ONFH.

\begin{tabular}{|c|c|c|c|c|c|c|c|}
\hline \multirow{2}{*}{ SNPs } & \multirow{2}{*}{ Subgroups } & \multicolumn{2}{|c|}{ Genotype(n) } & \multicolumn{4}{|c|}{ Allele(\%) } \\
\hline & & $\mathrm{AA} / \mathrm{AB} / \mathrm{BB}$ & $p^{\mathrm{a}}$ & OR & & & $p^{\mathrm{b}}$ \\
\hline \multirow[t]{8}{*}{ rs470154(T/G) } & Controls & $3 / 60 / 245$ & & & & & \\
\hline & Case-Unilateral & $4 / 15 / 64$ & 0.094 & 1.34 & 0.81 & 2.23 & 0.258 \\
\hline & Case-Bilateral & $5 / 52 / 146$ & 0.250 & 1.50 & 1.04 & 2.18 & $0.031 *$ \\
\hline & Case-Stage II & $3 / 11 / 50$ & 0.130 & 1.28 & 0.72 & 2.26 & 0.401 \\
\hline & Case-Stage III & $5 / 34 / 87$ & $0.015 *$ & 1.76 & 1.17 & 2.67 & $0.007 *$ \\
\hline & Case-Stage IV & $1 / 22 / 73$ & 0.820 & 1.19 & 0.72 & 1.96 & 0.492 \\
\hline & Case $>12$ months & $5 / 31 / 121$ & 0.190 & 1.25 & 0.83 & 1.90 & 0.290 \\
\hline & Case $\leq 12$ months & $4 / 36 / 89$ & 0.074 & 1.71 & 1.13 & 2.59 & $0.010 *$ \\
\hline \multirow[t]{8}{*}{ rs243866 (A/G) } & Controls & $8 / 68 / 231$ & & & & & \\
\hline & Case-Unilateral & $0 / 10 / 73$ & $0.013 *$ & 0.40 & 0.20 & 0.80 & $0.007 *$ \\
\hline & Case-Bilateral & $3 / 48 / 152$ & 0.470 & 0.97 & 0.67 & 1.40 & 0.862 \\
\hline & Case-Stage II & $1 / 10 / 53$ & 0.400 & 0.65 & 0.35 & 1.23 & 0.187 \\
\hline & Case-Stage III & $1 / 21 / 104$ & 0.240 & 0.63 & 0.39 & 1.03 & 0.064 \\
\hline & Case-Stage IV & $1 / 27 / 68$ & 0.240 & 1.12 & 0.71 & 1.77 & 0.620 \\
\hline & Case $>12$ months & $2 / 32 / 123$ & 0.470 & 0.82 & 0.54 & 1.24 & 0.341 \\
\hline & Case $\leq 12$ months & $1 / 26 / 102$ & 0.240 & 0.77 & 0.49 & 1.21 & 0.255 \\
\hline \multirow[t]{8}{*}{ rs243864 (G/T) } & Controls & $8 / 70 / 230$ & & & & & \\
\hline & Case-Unilateral & $0 / 10 / 73$ & $0.010 *$ & 0.40 & 0.20 & 0.78 & $0.006 *$ \\
\hline & Case-Bilateral & $3 / 48 / 152$ & 0.500 & 0.95 & 0.66 & 1.36 & 0.764 \\
\hline & Case-Stage II & $1 / 10 / 53$ & 0.360 & 0.64 & 0.34 & 1.20 & 0.163 \\
\hline & Case-Stage III & $1 / 21 / 104$ & 0.200 & 0.62 & 0.38 & 1.01 & 0.051 \\
\hline & Case-Stage IV & $1 / 27 / 68$ & 0.270 & 1.10 & 0.69 & 1.73 & 0.692 \\
\hline & Case $>12$ months & $2 / 32 / 123$ & 0.470 & 0.80 & 0.53 & 1.21 & 0.286 \\
\hline & Case $\leq 12$ months & $1 / 26 / 102$ & 0.230 & 0.75 & 0.48 & 1.18 & 0.213 \\
\hline \multirow[t]{8}{*}{ rs865094 (G/A) } & Controls & $22 / 120 / 166$ & & & & & \\
\hline & Case-Unilateral & $8 / 44$ / 31 & $0.029 *$ & 1.56 & 1.08 & 2.24 & $0.016 *$ \\
\hline & Case-Bilateral & $9 / 88 / 106$ & 0.430 & 0.97 & 0.73 & 1.29 & 0.855 \\
\hline & Case-Stage II & $4 / 30 / 30$ & 0.500 & 1.16 & 0.77 & 1.77 & 0.478 \\
\hline & Case-Stage III & $9 / 58 / 59$ & 0.780 & 1.19 & 0.86 & 1.64 & 0.290 \\
\hline & Case-Stage IV & $4 / 92 / 48$ & 0.370 & 1.02 & 0.71 & 1.47 & 0.900 \\
\hline & Case $>12$ months & $9 / 70 / 78$ & 0.750 & 1.07 & 0.79 & 1.45 & 0.649 \\
\hline & Case $\leq 12$ months & $8 / 62$ / 59 & 0.240 & 1.19 & 0.87 & 1.65 & 0.277 \\
\hline \multirow[t]{8}{*}{ rs11646643 (G/A) } & Controls & $11 / 104 / 193$ & & & & & \\
\hline & Case-Unilateral & $1 / 18$ / 64 & 0.062 & 0.53 & 0.32 & 0.88 & $0.014 *$ \\
\hline & Case-Bilateral & $5 / 70 / 127$ & 0.320 & 0.96 & 0.70 & 1.31 & 0.800 \\
\hline & Case-Stage II & $2 / 22 / 40$ & 0.640 & 0.99 & 0.62 & 1.59 & 0.971 \\
\hline & Case-Stage III & $2 / 38 / 86$ & 0.360 & 0.78 & 0.53 & 1.14 & 0.200 \\
\hline & Case-Stage IV & $2 / 28 / 65$ & 0.430 & 0.79 & 0.51 & 1.21 & 0.273 \\
\hline & Case $>12$ months & $4 / 44 / 108$ & 0.370 & 0.78 & 0.54 & 1.11 & 0.166 \\
\hline & Case $\leq 12$ months & $2 / 44 / 83$ & 0.320 & 0.89 & 0.61 & 1.29 & 0.532 \\
\hline \multirow[t]{8}{*}{ rs2241146 (A/G) } & Controls & 12 / $107 / 188$ & & & & & \\
\hline & Case-Unilateral & 10 / $30 / 43$ & $0.038 *$ & 1.59 & 1.08 & 2.33 & $0.017 *$ \\
\hline & Case-Bilateral & $13 / 58 / 132$ & 0.230 & 0.96 & 0.71 & 1.31 & 0.804 \\
\hline & Case-Stage II & $6 / 14 / 44$ & 0.076 & 0.94 & 0.59 & 1.51 & 0.797 \\
\hline & Case-Stage III & $7 / 44 / 75$ & 0.650 & 1.10 & 0.78 & 1.57 & 0.587 \\
\hline & Case-Stage IV & $10 / 30 / 56$ & 0.078 & 1.30 & 0.89 & 1.89 & 0.173 \\
\hline & Case $>12$ months & $17 / 51 / 89$ & $0.013 *$ & 1.37 & 1.00 & 1.88 & 0.050 \\
\hline & Case $\leq 12$ months & $6 / 37 / 86$ & 0.710 & 0.86 & 0.60 & 1.25 & 0.435 \\
\hline
\end{tabular}


TABLE 4: Continued.

\begin{tabular}{|c|c|c|c|c|c|c|c|}
\hline \multirow{2}{*}{ SNPs } & \multirow{2}{*}{ Subgroups } & \multicolumn{2}{|c|}{ Genotype(n) } & \multicolumn{4}{|c|}{ Allele(\%) } \\
\hline & & $\mathrm{AA} / \mathrm{AB} / \mathrm{BB}$ & $p^{\mathrm{a}}$ & OR & & & $p^{\mathrm{b}}$ \\
\hline \multirow[t]{8}{*}{ rs9928731 (T/C) } & Controls & $63 / 166 / 78$ & & & & & \\
\hline & Case-Unilateral & $17 / 43 / 23$ & 0.970 & 0.95 & 0.68 & 1.35 & 0.789 \\
\hline & Case-Bilateral & $47 / 107 / 49$ & 0.860 & 1.08 & 0.84 & 1.39 & 0.542 \\
\hline & Case-Stage II & $15 / 29 / 20$ & 0.550 & 0.94 & 0.64 & 1.38 & 0.763 \\
\hline & Case-Stage III & $28 / 72 / 26$ & 0.600 & 1.14 & 0.85 & 1.53 & 0.387 \\
\hline & Case-Stage IV & $21 / 49 / 26$ & 0.980 & 0.99 & 0.72 & 1.37 & 0.969 \\
\hline & Case $>12$ months & $27 / 88 / 42$ & 0.670 & 0.91 & 0.69 & 1.20 & 0.500 \\
\hline & Case $\leq 12$ months & 37 / 62 / 30 & 0.270 & 1.23 & 0.92 & 1.64 & 0.164 \\
\hline
\end{tabular}

$* P<0.05$ indicates statistical significance.

${ }^{a} P$ values were calculated by Wald test adjusted for age and gender.

${ }^{\mathrm{b}} P$ values were calculated by Pearson Chi-squared test.

receptor to this region was affected by differences in allelic expression [27]. Located in the GATA-1 site (CTATCT) of the promoter region of $M M P 2$, the rs 243866 polymorphism has binding sites for transcription factors AP-2, p53, Sp1, and Sp3. Protease expression is regulated by these transcription factors by the transcription rate of the gene [28]. For rs 243864 polymorphism, a paper reported that the rs243864, rs243865, 243866 haplotype was statistically significant related to childhood obesity [29]. In addition, rs243864 is present in the gene promoter, which is a tag SNP of the rs243865 and rs243866 polymorphisms [30]. It has been reported that genetic associations in the $M M P 2$ promoter may be caused by changes in MMP expression, because the three SNPs studied are functional SNPs that affect transcriptional activity.

Our study also found that MMP2 gene rs243866 and rs243864 may be the two protective SNPs of steroidinduced ONFH. The strong linkage between each pair of two SNPs (rs243866 and rs243864) confirmed that rs243866 and rs243864 could act not only alone but also coinitiate disease. In the Mexican mestizo population, rs243864 and rs11646643 of the MMP2 gene were associated with the high risk of COPD [28]. Our conclusion was that rs11646643 may be a low-risk factor of unilateral steroid-induced ONFH. And there was a strong linkage between each pair of the three SNPs (rs11646643, rs2241146, and rs9928731). But we did not find an association between steroid-induced ONFH and the above haplotype. In the study of Alicia Beeghly-Fadiel [31], rs2241146 may be unrelated to breast cancer risk. In this study, we found rs2241146 may be a high-risk factor of steroidinduced ONFH (especially unilateral ONFH).

Similarly, in our study, we found that rs470154 SNP in $M M P 10$ gene had an increased risk steroid-induced of ONFH (especially bilateral ONFH, clinical stage III, and $\leq 12$ months course). The other study showed that there is no association between rs470154 and the susceptibility to disc degeneration [32]. Information on specific genetic variants is inconsistent across different diseases, which can be explained by genetic differences between these diseases. It also illustrates the complexity of the mechanism involved in promoting SNP in ONFH. The differences observed here do not necessarily contradict previous reports. Further research is needed in populations with different ethnic and genetic backgrounds.
There were some important discoveries revealed in our study, but some limitations of this study should be considered when interpreting these results. First of all, our study did not include an analysis of biological functions, which will be crucial for elucidating the role of the gene in steroidinduced ONFH. Secondly, the sample size was relatively small, which might convert the positive findings into negative results. A larger case-control study is expected to circumvent those problems, which could make our conclusions more powerful. Although we obtained evidence for the association between $M M P 2$ and $M M P 10$ gene polymorphisms and steroid-induced ONFH risk, the pathogenesis of steroidinduced ONFH was still not completely understood. Further studies should be carried out to verify the present study in other ethnicity.

\section{Conclusions}

The MMP10 may be related to increased risk of steroidinduced ONFH. It is the first time that our results reveal an association between a MMP2 SNP at the rs2241146 locus and an increased risk of steroid-induced ONFH in Chinese Han population. And we found statistically significant differences in clinical phenotypes among the six SNPs (rs470154, rs243866, rs243864, rs865094, rs11646643, and rs2241146). From the genetic point of view to explore the pathogenesis of steroid-induced ONFH, early detection of susceptible population, timely intervention, and active prevention, the current treatment model can be transferred from postoperative surgery to predisease prevention. This study provides an important data source for the establishment of a bone necrosis gene bank in China. It can be used as a molecular marker for the diagnosis of osteonecrosis in the future, which not only benefits the majority of patients, but also has significant economic and social significance.

\section{Data Availability}

The data used to support the findings of this study are available from the corresponding author upon request. 


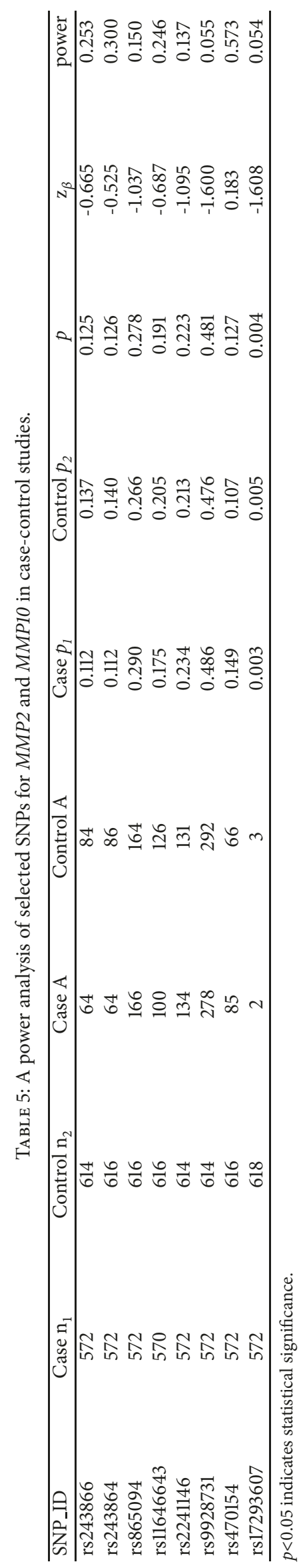


TABLE 6: The haplotype frequencies of $M M P 2$ polymorphisms and their association with steroid-induced ONFH risk.

\begin{tabular}{|c|c|c|c|c|c|}
\hline rs11646643 & $\begin{array}{c}\text { Haplotype } \\
\text { rs2241146 }\end{array}$ & rs9928731 & Freq & $\mathrm{OR}^{\mathrm{a}}(95 \% \mathrm{CI})$ & $p^{\mathrm{a}}$ \\
\hline A & $\mathrm{G}$ & $\mathrm{T}$ & 0.481 & 1 & - \\
\hline A & A & $\mathrm{C}$ & 0.220 & $1.13(0.84-1.54)$ & 0.420 \\
\hline G & G & $\mathrm{C}$ & 0.188 & $0.84(0.60-1.19)$ & 0.330 \\
\hline A & G & $\mathrm{C}$ & 0.108 & $0.86(0.57-1.29)$ & 0.470 \\
\hline
\end{tabular}

$p<0.05$ indicates statistical significance.

$\mathrm{a}=$ adjusted by gender and age.

TABLE 7: The haplotype frequencies of $M M P 2$ polymorphisms and their association with steroid-induced ONFH risk.

\begin{tabular}{|c|c|c|c|c|}
\hline \multicolumn{2}{|c|}{ Haplotype } & \multirow{2}{*}{ Freq } & \multirow{2}{*}{$\mathrm{OR}^{\mathrm{a}}(95 \% \mathrm{CI})$} & \multirow{2}{*}{$p^{\mathrm{a}}$} \\
\hline rs243866 & rs243864 & & & \\
\hline G & $\mathrm{T}$ & 0.874 & 1 & - \\
\hline A & G & 0.125 & $0.77(0.53-1.10)$ & 0.150 \\
\hline
\end{tabular}

\section{Ethical Approval}

Ethics committee approval was received for this study from Zhengzhou Traditional Chinese Medicine Traumatology Hospital.

\section{Consent}

All participants provided written informed consent.

\section{Disclosure}

Ye Tian and Feimeng An are co-first authors.

\section{Conflicts of Interest}

The authors declare there are no conflicts of interest.

\section{Authors' Contributions}

Jiaqi Wang and Chang Liu conceived and designed the experiments. Ye Tian and Feimeng An wrote the paper. Huiqiang $\mathrm{Wu}$ contributed reagents/materials/analysis tools. Yuju Cao recruited samples. All authors reviewed the manuscript.

\section{Acknowledgments}

We gratefully acknowledge all of the participants, the clinicians, and all the hospital staff for their contributions to this study. We are also thankful to Zhengzhou TCM Traumatology Hospital, Zhengzhou, Henan Province, China, for their support. This work received the support of the National Natural Science Foundation of China (nos. 81160228, 81260284, and 81660378).

\section{References}

[1] H. Kim, C. Cho, Y. Cho, S. Cho, K. Yoon, and K. Kim, "Significant associations of PAI-1 genetic polymorphisms with osteonecrosis of the femoral head," BMC Musculoskeletal Disorders, vol. 12, article 160, 2011.

[2] T.-H. Kim, J. M. Hong, H.-J. Kim, E. K. Park, and S.-Y. Kim, "Lack of association of MTHFR gene polymorphisms with the risk of osteonecrosis of the femoral head in a Korean population," Molecules and Cells, vol. 29, no. 4, pp. 343-348, 2010.

[3] Y. Song, Z. Du, M. Ren et al., "Association of gene variants of transcription factors PPAR $\gamma$, RUNX2, Osterix genes and COL2A1, IGFBP3 genes with the development of osteonecrosis of the femoral head in Chinese population," Bone, vol. 101, pp. 104-112, 2017.

[4] L. Cui, Q. Zhuang, J. Lin et al., "Multicentric epidemiologic study on six thousand three hundred and ninety five cases of femoral head osteonecrosis in China," International Orthopaedics, vol. 40, no. 2, pp. 267-276, 2016.

[5] G. Hadjigeorgiou, E. Dardiotis, M. Dardioti, A. Karantanas, A. Dimitroulias, and K. Malizos, "Genetic association studies in osteonecrosis of the femoral head: Mini review of the literature," Skeletal Radiology, vol. 37, no. 1, pp. 1-7, 2008.

[6] J. Du, W. Liu, T. Jin et al., "A single-nucleotide polymorphism in MMP9 is associated with decreased risk of steroid-induced osteonecrosis of the femoral head," Oncotarget, vol. 7, no. 42, pp. 68434-68441, 2016.

[7] G. Murphy and A. J. Docherty, "The matrix metalloproteinases and their inhibitors.", American Journal of Respiratory Cell and Molecular Biology, vol. 7, no. 2, pp. 120-125, 1992.

[8] S. L. Parsons, S. A. Watson, P. D. Brown, H. M. Collins, and R. J. C. Steele, "Matrix metalloproteinases," British Journal of Surgery, vol. 84, no. 2, pp. 160-166, 1997.

[9] K. Białkowska, W. Marciniak, M. Muszyńska et al., "Association of zinc level and polymorphism in MMP-7 gene with prostate cancer in Polish population," PLoS ONE, vol. 13, no. 7, Article ID e0201065, 2018.

[10] C. Kapoor, S. Vaidya, V. Wadhwan, Hitesh, G. Kaur, and A. Pathak, "Seesaw of matrix metalloproteinases (MMPs)," Journal of Cancer Research and Therapeutics, vol. 12, no. 1, pp. 28-35, 2016.

[11] J. S. Nyman, C. C. Lynch, D. S. Perrien et al., "Differential effects between the loss of MMP-2 and MMP-9 on structural and tissue-level properties of bone," Journal of Bone and Mineral Research, vol. 26, no. 6, pp. 1252-1260, 2011.

[12] K. Inoue, Y. Mikuni-Takagaki, K. Oikawa et al., "A crucial role for matrix metalloproteinase 2 in osteocytic canalicular formation and bone metabolism," The Journal of Biological Chemistry, vol. 281, no. 44, pp. 33814-33824, 2006. 
[13] S. Grässel, J. Beckmann, B. Rath, M. Vogel, J. Grifka, and M. Tingart, "Expression profile of matrix metalloproteinase-2 and -9 and their endogenous tissue inhibitors in osteonecrotic femoral heads," International Journal of Molecular Medicine, vol. 26, no. 1, pp. 127-133, 2010.

[14] J. A. Martignetti, A. A. Aqeel, W. A. Sewairi et al., "Mutation of the matrix metalloproteinase 2 gene (MMP2) causes a multicentric osteolysis and arthritis syndrome," Nature Genetics, vol. 28, no. 3, pp. 261-265, 2001.

[15] Y. Han, M. Si, Y. Zhao et al., "Progranulin protects against osteonecrosis of the femoral head by activating ERK1/2 pathway," Inflammation, vol. 40, no. 3, pp. 946-955, 2017.

[16] M. Onishi, T. Kobayashi, C. N. D’Alessandro-Gabazza et al., "Mice overexpressing latent matrix metalloproteinase-2 develop lung emphysema after short-term exposure to cigarette smoke extract," Biochemical and Biophysical Research Communications, vol. 497, no. 1, pp. 332-338, 2018.

[17] A. Purroy, C. Roncal, J. Orbe et al., "Matrix metalloproteinase10 deficiency delays atherosclerosis progression and plaque calcification," Atherosclerosis, vol. 278, pp. 124-134, 2018.

[18] S. N. Pandruvada, O. A. Gonzalez, S. Kirakodu et al., "Bone biology-related gingival transcriptome in ageing and periodontitis in non-human primates," Journal of Clinical Periodontology, vol. 43, no. 5, pp. 408-417, 2016.

[19] F. H. Duits, M. Hernandez-Guillamon, J. Montaner et al., "Matrix metalloproteinases in alzheimer's disease and concurrent cerebral microbleeds," Journal of Alzheimer's Disease, vol. 48, no. 3, pp. 711-720, 2015.

[20] Y. Yu, Z. Xie, J. Wang et al., "Single-nucleotide polymorphisms of MMP2 in MMP/TIMP pathways associated with the risk of alcohol-induced osteonecrosis of the femoral head in Chinese males: A case-control study," Medicine (United States), vol. 95, no. 49, p. e5407, 2016.

[21] Q. Wang, Q. Yang, G. Chen et al., "LncRNA expression profiling of BMSCs in osteonecrosis of the femoral head associated with increased adipogenic and decreased osteogenic differentiation," Scientific Reports, vol. 8, no. 1, 2018.

[22] R. Wojciechowski, J. E. Bailey-Wilson, and D. Stambolian, "Association of matrix metalloproteinase gene polymorphisms with refractive error in amish and ashkenazi families," Investigative Ophthalmology \& Visual Science, vol. 51, no. 10, pp. 49894995, 2010.

[23] O. Liu, J. Li, Y. Xin et al., "Association of MMP-2 gene haplotypes with thoracic aortic dissection in chinese han population," $B M C$ Cardiovascular Disorders, vol. 16, no. 1, p. 11, 2016.

[24] R. Wojciechowski, S. S. Yee, C. L. Simpson, J. E. BaileyWilson, and D. Stambolian, "Matrix metalloproteinases and educational attainment in refractive error: Evidence of geneenvironment interactions in the age-related eye disease study," Ophthalmology, vol. 120, no. 2, pp. 298-305, 2013.

[25] N. A. Juiz, N. M. Cayo, M. Burgos et al., "Human polymorphisms in placentally expressed genes and their association with susceptibility to congenital trypanosoma cruzi infection," The Journal of Infectious Diseases, vol. 213, no. 8, pp. 1299-1306, 2016.

[26] Y. Hua, N. Wu, Y. Yang et al., "Polymorphisms of MMP-2 gene are associated with systolic heart failure risk in han chinese," The American Journal of the Medical Sciences, vol. 337, no. 5, pp. 344348, 2009.

[27] S. Harendza, D. H. Lovett, U. Panzer, Z. Lukacs, P. Kühnl, and R. A. K. Stahl, "Linked common polymorphisms in the gelatinase a promoter are associated with diminished transcriptional response to estrogen and genetic fitness," The Journal of Biological Chemistry, vol. 278, no. 23, pp. 20490-20499, 2003.

[28] J. Hernández-Montoya, J. Pérez-Ramos, M. Montaño et al., "Genetic polymorphisms of matrix metalloproteinases and protein levels in chronic obstructive pulmonary disease in a Mexican population," Biomarkers in Medicine, vol. 9, no. 10, pp. 979-988, 2015.

[29] A. R. Morgan, D. Y. Han, J. M. Thompson, E. A. Mitchell, and L. R. Ferguson, "Analysis of MMP2 promoter polymorphisms in childhood obesity," BMC Research Notes, vol. 4, no. 1, 2011.

[30] I. Ito, S. Nagai, T. Handa et al., "Matrix metalloproteinase-9 promoter polymorphism associated with upper lung dominant emphysema," American Journal of Respiratory and Critical Care Medicine, vol. 172, no. 11, pp. 1378-1382, 2005.

[31] A. Beeghly-Fadiel, W. Lu, J.-R. Long et al., "Matrix metalloproteinase-2 polymorphisms and breast cancer susceptibility," Cancer Epidemiol Biomarkers Prev, vol. 18, no. 6, pp. 1770-1776, 2009.

[32] A. Kelempisioti, P. J. Eskola, A. Okuloff et al., "Genetic susceptibility of intervertebral disc degeneration among young Finnish adults," BMC Medical Genetics, vol. 12, no. 1, 2011. 


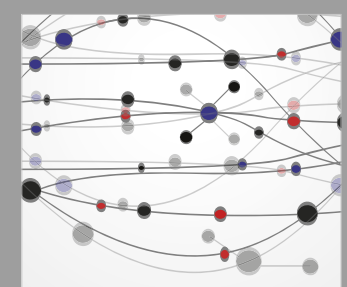

The Scientific World Journal
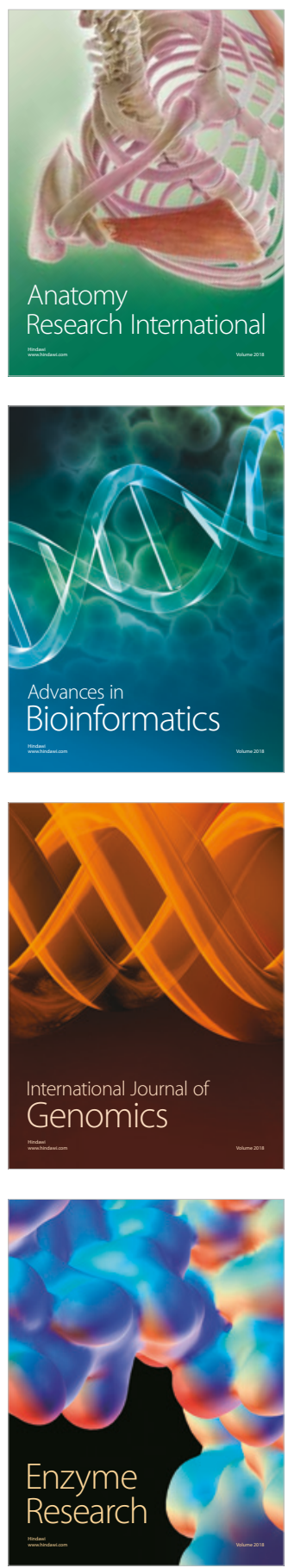
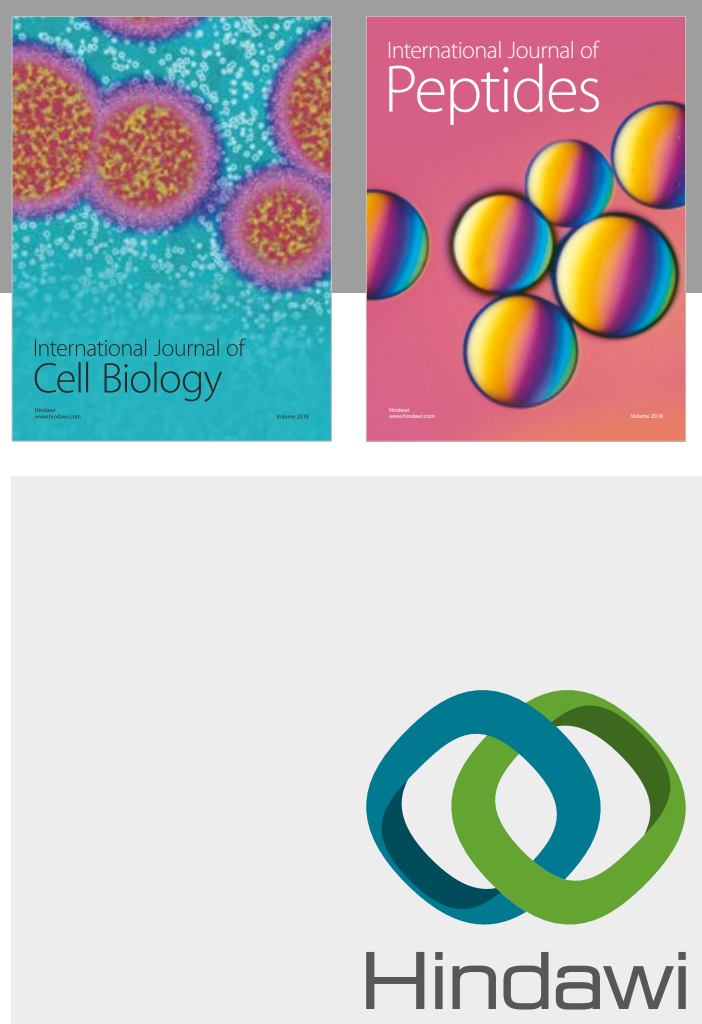

Submit your manuscripts at

www.hindawi.com
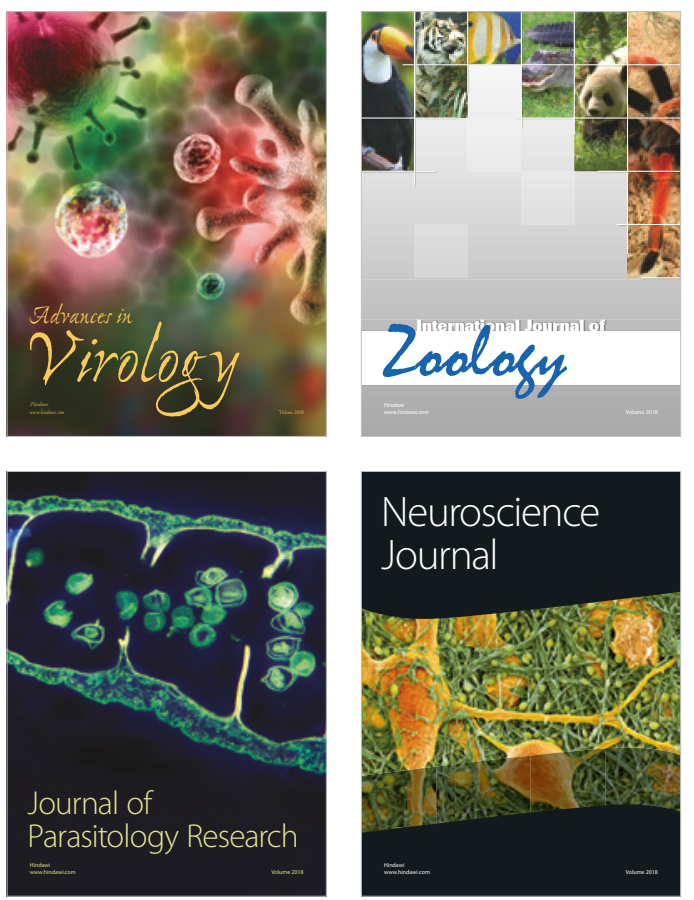
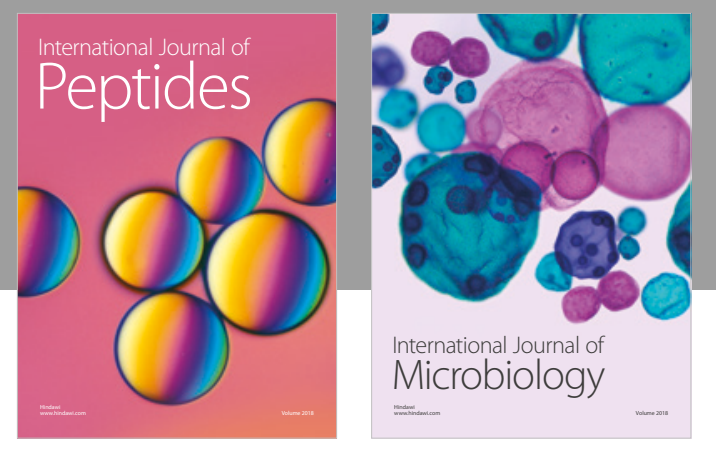

nternational Journal of Microbiology
Journal of
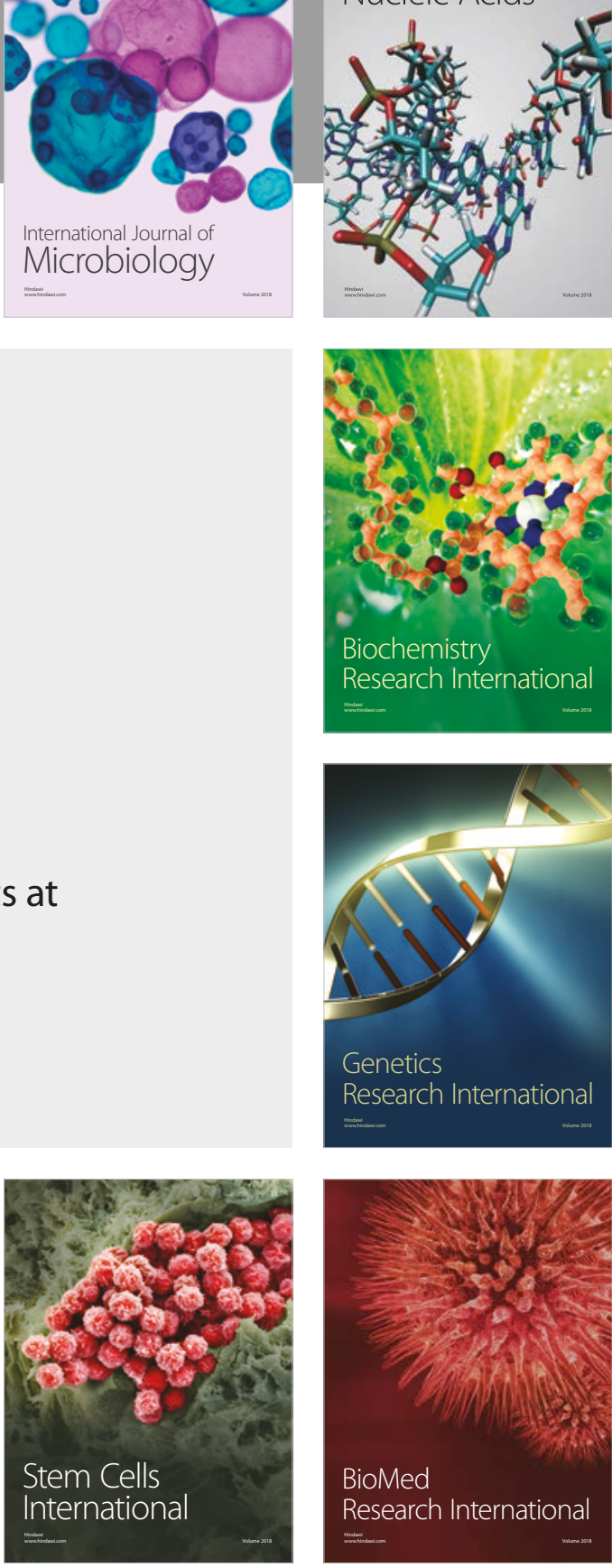
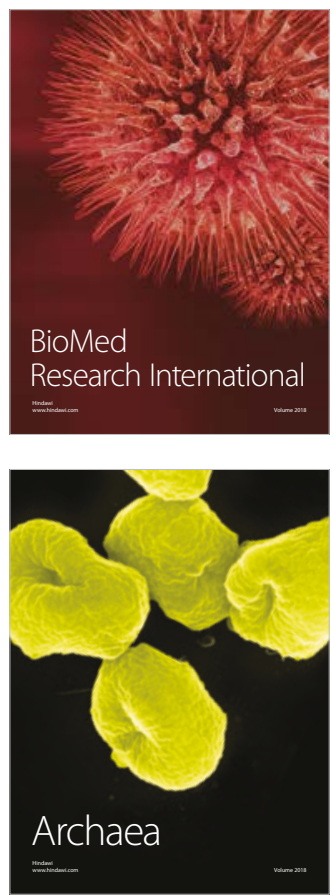